\title{
Imaging Microglial/Macrophage Activation in Spinal Cords of Experimental Autoimmune Encephalomyelitis Rats by Positron Emission Tomography Using the Mitochondrial 18 $\mathrm{kDa}$ Translocator Protein Radioligand $\left[{ }^{18} \mathrm{~F}\right] \mathrm{DPA}-714$
}

\author{
Galith Abourbeh, ${ }^{1,2}$ Benoit Thézé, ${ }^{1,2}$ Renaud Maroy, ${ }^{2}$ Albertine Dubois, ${ }^{1,2}$ Vincent Brulon, ${ }^{2}$ Yoann Fontyn, ${ }^{2}$ \\ Frédéric Dollé, ${ }^{2}$ Bertrand Tavitian, ${ }^{1,2}$ and Raphaël Boisgard ${ }^{1,2}$ \\ ${ }^{1}$ Inserm, Unité 1023, Université Paris Sud, 91400 Orsay, France, and ${ }^{2}$ Commissariat à l'Énergie Atomique et aux Énergies Alternatives, Direction des \\ Sciences du Vivant, Institut d'Imagerie Biomédicale, Service Hospitalier Frédéric Joliot, Laboratoire d’Imagerie Moléculaire Expérimentale, 91405 Orsay, France
}

\begin{abstract}
Multiple sclerosis (MS) is an inflammatory demyelinating disease of the CNS. Activated microglia/macrophages play a key role in the immunopathogenesis of MS and its corresponding animal models, experimental autoimmune encephalomyelitis (EAE). Microglia activation begins at early stages of the disease and is associated with elevated expression of the $18 \mathrm{kDa}$ mitochondrial translocator protein (TSP0). Thus, positron emission tomography (PET) imaging of microglial activation using TSPO-specific radioligands could be valuable for monitoring disease-associated neuroinflammatory processes. EAE was induced in rats using a fragment of myelin basic protein, yielding acute clinical disease that reflects extensive spinal cord inflammation. Enhanced TSPO expression in spinal cords of EAE rats versus those of controls was confirmed by Western blot and immunohistochemistry. Biodistribution studies in control and EAE rats were performed using the TSPO radioligand $\left[{ }^{18} \mathrm{~F}\right] \mathrm{DPA}-714$ [N,N-diethyl-2-(2-(4-(2-fluoroethoxy)phenyl)-5,7-dimethylpyrazolo[1,5a]pyrimidin-3-yl)acetamide]. At $1 \mathrm{~h}$ after injection, almost fivefold higher levels of $\left[{ }^{18} \mathrm{~F}\right] \mathrm{DPA}-714$ were measured in spinal cords of EAE rats versus controls. The specific binding of $\left[{ }^{18} \mathrm{~F}\right] \mathrm{DPA}-714$ to TSPO in spinal cords was confirmed in competition studies, using unlabeled $(R, S)$-PK11195 [(R,S)-N-methyl- $N$-(1-methylpropyl)-1-(2-chlorophenyl)isoquinoline-3-carboxamide)] or DPA-714 in excess. MicroPET studies affirm that this differential radioactivity uptake in spinal cords of EAE versus control rats could be detected and quantified. Using $\left[{ }^{18} \mathrm{~F}\right] \mathrm{DPA}-714$, neuroinflammation in spinal cords of EAE-induced rats could be visualized by PET, offering a sensitive technique for monitoring neuroinflammatory lesions in the CNS and particularly in the spinal cord. In addition to current MRI protocols, this approach could provide molecular images of neuroinflammation for detection, monitoring, and research in MS.
\end{abstract}

\section{Introduction}

Multiple sclerosis (MS) is an inflammatory, demyelinating disease of the CNS that develops as a relapsing remitting, primary progressive, or secondary progressive disease. Current immunomodulatory and immunosuppressive treatments reduce the frequency of relapses and delay the time to progression to disabling

Received June 9, 2011; revised Jan. 3, 2012; accepted Jan. 6, 2012.

Author contributions:G.A., B.Th., and R.B. performed research;G.A., B.Th, and R.B. analyzed data;G.A., B.Ta., and R.B. designed research; R.M., A.D., V.B., Y.F., F.D., and B.Ta. contributed unpublished reagents/analytic tools; G.A. and R.B. wrote the paper.

This work was supported by the FP6 European Networks of Excellence EMIL (Grant LSHC-CT-2004-503569) and DiMI (Grant LSHB-CT-2005-512146), promoted by the European Community (RATstream STREP, Grant LSHM-CT2007-037846) and more recently the European Union's Seventh Framework Programme [FP7/2007-2013] INMiND (Grant agreement no. 278850). We thank Karine Siquier-Pernet and Benoit Jego for their technical assistance and Prof. Higuchi (The National Institute of Radiological Sciences, Chiba, Japan) for providing the rabbit polyclonal anti-TSPO antibody. We also express special appreciation to Dr. Doron Merkler (Department of Pathology and Immunology, University of Geneva, Geneva, Switzerland) for his advice and assistance in IHC staining.

Correspondence should be addressed to Raphaël Boisgard, Commissariat à l'Énergie Atomique et aux Énergies Alternatives, Direction des Sciences du Vivant, Institut d'Imagerie Biomédicale, Service Hospitalier Frédéric Joliot, Laboratoire d'Imagerie Moléculaire Expérimentale, Inserm, Unité 1023, 4, Place Géneral Leclerc, 91406 Orsay, France. E-mail: raphael.boisgard@cea.fr.

DOI:10.1523/JNEUROSCI.2900-11.2012

Copyright $\odot 2012$ the authors $\quad 0270-6474 / 12 / 325728-09 \$ 15.00 / 0$ stages in the relapsing remitting and secondary progressive forms, respectively. Therapeutic strategies aiming at neuroprotection, repair, and prevention of disease progression are under active exploration (Lopez-Diego and Weiner, 2008; Barkhof et al., 2009).

To date, MS diagnosis and monitoring is based on clinical evaluation and on magnetic resonance imaging (MRI). $\mathrm{T}_{2}$-MRI and gadolinium $(\mathrm{Gd})$-enhanced $\mathrm{T}_{1}$-MRI are highly sensitive in identifying tissue pathologies and allow separation of active and inactive lesions. Unfortunately, $\mathrm{T}_{2}$-MRI lacks specificity in distinguishing different histopathological findings typical of MS, leaving a need for an improved non-invasive technique that can provide more specific information concerning the underlying histolopathological processes. Furthermore, less conspicuous pathological events, such as subtle blood-brain barrier (BBB) alterations and diffuse white matter (WM) inflammation, cannot always be detected by Gd-enhancement (Kutzelnigg et al., 2005). Combining multiple imaging strategies is therefore invaluable for non-invasive, in vivo characterization of MS lesions. Multimodal imaging could not only improve diagnosis and monitoring in MS but could also have prognostic and therapeutic significance. 
Positron emission tomography (PET) provides a sensitive means for visualizing MS-related processes at the molecular level. Imaging neuroinflammation and CNS myelin by PET has been described in animal models using $2-\left[{ }^{18} \mathrm{~F}\right]$-fluoro-2-deoxyD-glucose and [ $\left.{ }^{11} \mathrm{C}\right]$ 1,4-bis ( $p$-aminostyryl)-2-methoxy benzene, respectively (Stankoff et al., 2006; Radu et al., 2007). Notably also, radiolabeled ligands that target the mitochondrial $18 \mathrm{kDa}$ translocator protein (TSPO) have been used extensively to image neuroinflammation by PET (Banati, 2002; Dollé et al., 2009; Winkeler et al., 2010). Increased expression of TSPO by activated microglia/macrophages in the CNS has been reported in various neuroinflammatory conditions and has been recognized as a hallmark of neuroinflammation (Banati, 2002, 2003; Turkheimer et al., 2004). Notably, histological and imaging findings indicate that activated microglia are strongly implicated in different types of MS lesions, including inactive chronic and cortical lesions, and in the normally appearing WM (NAWM) (Kutzelnigg et al., 2005; DuboisDalcq et al., 2008). Consequently, imaging microglial activation by PET using radiolabeled TSPO ligands should provide a sensitive marker of neuroinflammatory processes.

With regards to in vivo imaging of the CNS, spinal cord imaging is of particular challenge. Spinal cord lesions are prevalent in early-stage MS and often develop independently of brain lesions. Moreover, because much of the physical disability related to MS is also associated with spinal cord lesions, spinal cord imaging represents a field of considerable interest (Bot et al., 2004; Agosta et al., 2007; Ciccarelli et al., 2007).

The present work is a first demonstration of the feasibility of imaging neuroinflammatory spinal cord lesions in a rat model of MS, using the PET TSPO radioligand $\left[{ }^{18} \mathrm{~F}\right] \mathrm{DPA}-714[N, N$-diethyl2-(2-(4-(2-fluoroethoxy)phenyl)-5,7-dimethylpyrazolo[1,5a]pyrimidin-3-yl)acetamide]. Our results corroborate previous data demonstrating the value of $\left[{ }^{18} \mathrm{~F}\right] \mathrm{DPA}-714$ for imaging microglial activation in the CNS, in general, and extend the use of this approach to the imaging of spinal cord inflammation.

\section{Materials and Methods}

Induction of experimental autoimmune encephalomyelitis in rats. Animal studies were conducted in accordance with the French legislation and European directives on the use of animals in research. Female Lewis rats (Centre d'Elvage René Janvier) aged 7 weeks were acclimated in the animal facility for 1 week before experimental autoimmune encephalomyelitis (EAE) induction. Animals were kept in a thermoregulated, humidity-controlled environment, in $12 \mathrm{~h}$ light/dark cycles, and provided with food and water ad libitum.

For each of the subsequent experimental procedures (i.e., preparation of spinal cord tissue extracts, biodistribution, and imaging studies), EAE was induced in a separate group of animals. When possible, rats of one experimental group were also assigned to other experimental groups. Before each immunization, a myelin basic protein (MBP) emulsion was freshly prepared using a guinea pig (gp) $\mathrm{MBP}$ fragment $\left(\mathrm{MBP}_{68-86}\right.$, AnaSpec Inc., San Jose, CA, USA), as described previously (Stepaniak et al., 1995). Emulsification was performed by mixing an $\mathrm{MBP}_{68-86}$ solution $(0.5 \mathrm{mg} / \mathrm{ml}$ in PBS) with an equal volume of complete Freund's adjuvant (CFA), containing $4 \mathrm{mg} / \mathrm{ml}$ heat-inactivated Mycobacterium tuberculosis H37Ra (Difco Laboratories). Similarly, a control emulsion was freshly prepared by emulsifying equal volumes of PBS and CFA containing $4 \mathrm{mg} / \mathrm{ml}$ heat-inactivated $M$. tuberculosis. Both emulsions were kept overnight at $4^{\circ} \mathrm{C}$ until injection on the following day.

Immunization of rats was performed under anesthesia (3.0\% isoflurane in $\mathrm{O}_{2}$ ), by injecting $100 \mu \mathrm{l}$ of emulsion, divided equally between the hind footpads. Control rats were injected with an identical volume of a PBS/CFA emulsion.

Neurological assessment. Subsequent to each immunization, animal weight and neurological deficits were monitored daily, for $16 \mathrm{~d}$. The following scoring system was used to grade neurological impairment: 0 , no symptoms; 1 , flaccid tail; 2 , hindlimb weakness; 3 , paraparesis; 3.5 , unilateral hindlimb paralysis; 4 , bilateral hindlimb paralysis; and 5, bilateral hindlimb paralysis and incontinence.

Preparation of spinal cord tissue extracts. Spinal cord tissue extracts were prepared from EAE rats with a score of $4-5(n=7)$ and controls $(n=6)$. Before the excision of spinal cords, rats were deeply anesthetized with sodium thiopental $(60 \mathrm{mg} / \mathrm{kg}$, i.p.) and perfused transcardially with cold $\left(4^{\circ} \mathrm{C}\right)$ PBS. After decapitation, whole spinal cords were removed, snap frozen, and kept at $-70^{\circ} \mathrm{C}$ until additional processing. To enrich TSPO content in the tissue preparation, the excised spinal cords were homogenized in cold $\left(0-4^{\circ} \mathrm{C}\right)$, modified RIPA buffer, consisting of 50 $\mathrm{mm}$ Tris, $\mathrm{pH} 7.5,1 \%(\mathrm{v} / \mathrm{v}) \mathrm{NP}-40,0.5 \%(\mathrm{w} / \mathrm{v})$ deoxycholate, $50 \mathrm{~mm}$ sodium pyrophosphate, $100 \mathrm{~mm} \mathrm{NaF}, 5 \mathrm{~mm}$ sodium orthovanadate, and $150 \mathrm{~mm} \mathrm{NaCl}$. A protease inhibitor cocktail for mammalian tissues (P8340; Sigma) was added to the cold RIPA buffer at a ratio of 1:100, as recommended by the manufacturer. The homogenates were transferred to microcentrifuge tubes and centrifuged $\left(20 \mathrm{~min}, 20,000 \times \mathrm{g}, 4^{\circ} \mathrm{C}\right)$. Supernatants were separated and kept at $-20^{\circ} \mathrm{C}$ until additional analysis.

Western blot analysis. Determination of protein concentration in samples was made using Bradford protein assay (Bradford, 1976). Tissue extracts were mixed with boiling Laemli's buffer (Cleveland et al., 1977), and equal amounts of each sample (60 $\mu \mathrm{g}$ of total protein) were loaded and separated by SDS-PAGE (15\%). Proteins were electrophoretically transferred to a nitrocellulose membrane, and the latter was blocked in $3 \%$ BSA in TBST buffer (50 mm Tris-HCl, pH 7.5, 0.1\% Tween 20, and $150 \mathrm{~mm} \mathrm{NaCl}$ ) for $30 \mathrm{~min}$. Corresponding parts of the membrane were incubated overnight $\left(4^{\circ} \mathrm{C}\right.$ with gentle shake) with the following primary antibodies, diluted in $0.1 \%$ BSA/TBST or $5 \%$ low-fat milk/TBST, respectively: (1) rabbit anti-TSPO polyclonal antibody NP155 (1:15,000 dilution; kindly provided by Prof. Higuchi, National Institute of Radiological Sciences, Chiba, Japan) (Ji et al., 2008) and (2) mouse anti- $\alpha$-tubulin monoclonal antibody (1:40,000 dilution; B-5-1-2; Sigma). The membrane parts were washed thoroughly with TBST buffer and incubated for $1 \mathrm{~h}$ with the corresponding horseradish peroxidase-conjugated IgGs (1:2000 dilution in $0.1 \%$ BSA/TBST; Santa Cruz Biotechnology). Finally, the membranes were washed in TBST, and immunoreactive proteins were visualized using Amersham ECL Advance Western Blotting Detection (GE Healthcare). Densitometry was performed using NIH ImageJ 1.38x image processing program (National Institutes of Health), and the intensity of each TSPO band was normalized to that of the corresponding $\alpha$-tubulin band to correct for differences in the content of total loaded protein.

Immunohistochemistry. EAE rats (scores $4-5, n=5)$ and controls $(n=$ 2) were anesthetized with thiopental $(60 \mathrm{mg} / \mathrm{kg})$ and perfused transcardially with saline, followed by fixative containing $4 \% 0.1 \mathrm{~m}$ sodium phosphate-buffered paraformaldehyde, $\mathrm{pH}$ 7.4. Spinal cords were dissected, postfixed overnight in fresh fixative, and embedded in paraffin. A total of six paraffin tissue blocks comprising 25 spinal cord sections were used for histological evaluation. Immunohistochemistry (IHC) was performed on 5- $\mu \mathrm{m}$-thick sections, after deparaffinization and antigen unmasking by water-bath treatment in PT Module buffer, $\mathrm{pH} 8.0\left(95^{\circ} \mathrm{C}, 20\right.$ min +20 min cooling; Thermo Fisher Scientific). Sections were blocked with $5 \%$ BSA and $0.5 \%$ Tween 20 in PBS [ 5 min, room temperature (RT)] and incubated ( $1 \mathrm{~h}, \mathrm{RT}$ ) with primary antibodies as follows: (1) for case 1, chicken anti-glial fibrillary acid protein (GFAP) (ab4674, 1:500; Abcam), rabbit anti-TSPO antibody (NP155, 1:500) and mouse antiCD68 (MCA341R, 1:100; Serotec), diluted in 5\% BSA and 0.5\% Tween 20 in PBS, and, after PBS washes (three times), sections were incubated (30 min, RT) with Alexa Fluor-647 goat anti-chicken (A21449, 1:1000; Invitrogen), Alexa Fluor-488 goat anti-rabbit (A11034, 1:1000; Invitrogen), and Alexa Fluor-594 goat anti-mouse (A11032, 1:1000; Invitrogen), respectively, diluted in 5\% BSA and 0.5\% Tween 20 in PBS; (2) for case 2, goat anti-Iba-1 (ab5076; 1:100; Abcam), rabbit anti-TSPO antibody (NP155, 1:500) and mouse anti-CD68 (MCA341R, 1:100; Serotec), diluted in 5\% BSA and 0.5\% Tween 20 in PBS, and after PBS washes (three times), sections were incubated (30 min, RT) with Alexa Fluor-647 donkey anti-goat (A21447, 1:1000; Invitrogen), Alexa Fluor-488 donkey anti-rabbit (A21206, 1:1000; Invitrogen), and Alexa Fluor-546 donkey anti-mouse (A10036, 1:1000; Invitrogen), respectively, diluted in 5\% 
BSA and 0.5\% Tween 20 in PBS. After three additional washes, sections were mounted with a DAPI Prolong Antifade Kit (P36931; Invitrogen).

Chemistry and radiochemistry. Racemic PK11195 [(R,S)-N-methyl- $N-(1-$ methylpropyl)-1-(2-chlorophenyl)isoquinoline-3-carboxamide)] was kindly provided by Sanofi-Aventis. DPA-714 and its tosyloxy analog $[\mathrm{N}, \mathrm{N}-$ diethyl-2-(2-(4-(2-toluenesulfonyloxyethoxy)phenyl)-5,7-dimethylpyrazolo[1,5-a]pyrimidin-3-yl)acetamide] as precursor for fluorine-18 labeling were synthesized in two steps from DPA-713, according to procedures described previously (Damont et al., 2008). Solutions of $(R, S)$-PK11195 for intravenous injection were prepared by dissolving 2-3 mg of the compound in $0.2 \mathrm{ml}$ of dimethylsulfoxide (DMSO), followed by dilution with $1 \mathrm{ml}$ of polyethylene glycol 400 (PEG 400) and addition of $2 \mathrm{ml}$ of physiological saline. Similarly, solutions of unlabeled DPA-714 for intravenous injection were prepared by dissolving 2-3 mg in $0.2 \mathrm{ml}$ of DMSO, followed by dilution with $0.4 \mathrm{ml} \mathrm{PEG} 400$ and $1.2 \mathrm{ml}$ of physiological saline. Fresh solutions were routinely formulated before each in vivo challenge study.

Radiosynthesis of $\left[{ }^{18} \mathrm{~F}\right] \mathrm{DPA}-714$. DPA-714 was labeled with fluorine-18 (half-life, $109.8 \mathrm{~min}$ ) at its 2-fluoroethylmoiety using a tosyloxy-for-fluorine nucleophilic aliphatic substitution and with slight modifications with respect to a previously published synthesis (James et al., 2008). This one-step synthesis had been automated using a Zymate-XP robotic system (Damont et al., 2008) and applied to a commercially available GE TRACERLab FX-FN synthesizer (Kuhnast et al., 2012). In brief, the synthesis included (1) reaction of $\mathrm{K}\left[{ }^{18} \mathrm{~F}\right] \mathrm{F}$ Kryptofix222 with the tosyloxy precursor (4.5-5.0 mg, 8.2-9.1 $\mu \mathrm{mol})$ at $165^{\circ} \mathrm{C}$ for $5 \mathrm{~min}$ in DMSO $(0.6 \mathrm{ml})$, followed by (2) C-18 PrepSep cartridge (Thermo Fisher Scientific) prepurification, and (3) semipreparative HPLC purification on a Waters X-TerraRP18. A formulation of $\left[{ }^{18} \mathrm{~F}\right] \mathrm{DPA}-714$ for intravenous injection, containing $<10 \%$ ethanol in physiological saline, was prepared using a homemade SepPakPlus C18 cartridge. Typically, 5.6-7.4 GBq of $\left[{ }^{18} \mathrm{~F}\right] \mathrm{DPA}-714$ (>95\% chemically and radiochemically pure), with specific radioactivities ranging between 37 and $111 \mathrm{GBq} / \mu \mathrm{mol}$, were obtained within $\sim 90 \mathrm{~min}$ of radiosynthesis (HPLC purification and SepPak-based formulation included); starting from a $37 \mathrm{GBq}\left[{ }^{18} \mathrm{~F}\right]$ fluoride batch (overall non-decay-corrected, isolated radiochemical yield, $15-20 \%$ ).

Biodistribution studies. The distribution of $\left[{ }^{18} \mathrm{~F}\right] \mathrm{DPA}-714$ was evaluated in EAE-induced female Lewis rats with clinical scores ranging from 4 to 5 and in CFA-injected matched controls. For this purpose, 10-12 d after EAE-induction, MBP-injected rats $(150 \pm 2 \mathrm{~g}, n=30)$ and CFA-injected rats $(181 \pm 2 \mathrm{~g}, n=16)$ were administered with $\left[{ }^{18} \mathrm{~F}\right] \mathrm{DPA}-714$ (262 MBq/ $2.25 \pm 0.33 \mathrm{nmol} / \mathrm{rat})$. Rats were kept under anesthesia (1.5-3.0\% isoflurane in $\mathrm{O}_{2}$ ) during $1 \mathrm{~h}$ after injection of the radiotracer, after which they were deeply anesthetized with sodium thiopental $(60 \mathrm{mg} / \mathrm{kg}$, i.p.). Next, blood samples were collected, rats were perfused transcardially with PBS, and decapitated, and spinal cords were excised. Blood and tissue samples were weighed, and radioactivity was counted in a gamma counter (Cobra II AutoGamma; PerkinElmer Life and Analytical Sciences). Distribution of radioactivity was decay corrected to the time of $\left[{ }^{18} \mathrm{~F}\right] \mathrm{DPA}-714$ injection and calculated as the percentage of the total injected dose per gram of wet tissue $(\% \mathrm{ID} / \mathrm{g})$. For $\left[{ }^{18} \mathrm{~F}\right] \mathrm{DPA}-714$ displacement studies, an excess of either of the following unlabeled compounds was used: $(1)(R, S)$-PK11195 (1.09 \pm 0.04 $\mathrm{mg} / \mathrm{kg}, n=8)$ or (2) DPA-714 (1.13 $\pm 0.06 \mathrm{mg} / \mathrm{kg}, n=7)$. Injections of $\left[{ }^{18} \mathrm{~F}\right] \mathrm{DPA}-714$ and of the unlabeled challenge compounds were made in the caudal lateral vein using a 24 gauge catheter. Unlabeled compounds were administered $30 \mathrm{~min}$ after $\left[{ }^{18} \mathrm{~F}\right] \mathrm{DPA}-714$ injection.

PET scans and data acquisition. PET scans were performed 10-12 d after EAE/CFA injection in EAE-induced female Lewis rats with clinical scores ranging from 4 to $5(148 \pm 2 \mathrm{~g}, n=23)$ and in CFA-injected matched controls ( $176 \pm 3 \mathrm{~g}, n=8)$. Anesthesia was induced, and thereafter maintained, by 3 and $1-2.5 \%$ isoflurane in $\mathrm{O}_{2}$, respectively. During the scans, rats were maintained normothermic using a heating blanket (Homeothermic Blanket Control Unit; Harvard Apparatus). For optimal visualization of the spinal cord, the dorsal side of the rats, comprising the thoracic and lumbar parts of the spinal column, was positioned along the midsagittal axis of the PET field of view (FOV). Dynamic PET data acquisition was performed using a Focus 220 PET scanner (Siemens Medical Solutions). Imaging was started at the time of injection of $\left[{ }^{18} \mathrm{~F}\right] \mathrm{DPA}-714(38 \pm 1 \mathrm{MBq} / 3.07 \pm 0.45 \mathrm{nmol} / \mathrm{rat}, n=31)$ and was continued for $68 \mathrm{~min}$ after injection of the radiotracer. Competition for $\left[{ }^{18} \mathrm{~F}\right] \mathrm{DPA}-714$ uptake was performed by injecting an excess of either of the following unlabeled compounds into a subset of the EAE rats: (1) $(R, S)-P K 11195(1.23 \pm 0.09 \mathrm{mg} / \mathrm{kg}, n=8)$ or $(2) \mathrm{DPA}-714(1.13 \pm 0.06$ $\mathrm{mg} / \mathrm{kg}, n=7)$. Injections of $\left[{ }^{18} \mathrm{~F}\right] \mathrm{DPA}-714$ and of the unlabeled challenge compounds were all made in the caudal lateral vein using a 24 gauge catheter. Radiolabeled DPA-714 was injected concomitantly with the start of PET acquisition, and unlabeled compounds were administered $30 \mathrm{~min}$ after injection of the radiotracer.

For delineation of the spinal column, in a selected number of studies $(n=12)$, the $\left[{ }^{18} \mathrm{~F}\right] \mathrm{DPA}-714$ PET acquisition was immediately followed by a $20 \mathrm{~min}\left[{ }^{18} \mathrm{~F}\right]$ fluoride scan, without moving the rat from the PET bed. Specifically, at the end of the $\left[{ }^{18} \mathrm{~F}\right] \mathrm{DPA}-714 \mathrm{scan},\left[{ }^{18} \mathrm{~F}\right]$ fluoride $(36 \pm 2$ $\mathrm{MBq} / \mathrm{rat}$ ) was injected in the lateral tail vain, and $5 \mathrm{~min}$ later, a second PET scan was acquired during $20 \mathrm{~min}$.

Acquisitions were conducted with a time coincidence window of $6 \mathrm{~ns}$, and the levels of energy discrimination were set to 350 and $650 \mathrm{KeV}$. The list-mode acquisition data files were histogrammed into threedimensional sinograms with a maximum ring difference of 47 and a span of 3. The list-mode data of $\left[{ }^{18} \mathrm{~F}\right] \mathrm{DPA}-714$ emission scans were sorted into 24 (or 31 for competition studies) dynamic frames, and those of $\left[{ }^{18} \mathrm{~F}\right]$ fluoride emission scans were sorted into a single static frame. The attenuation correction factors were measured using an external ${ }^{68} \mathrm{Ge}$ point source. Finally, the emission sinograms (each frame) were normalized and corrected for attenuation, scattering, and radioactivity decay. In an effort to get better resolution of the spinal cord, image reconstruction was performed using Fourier rebinning and both of the following reconstruction algorithms: (1) two-dimensional ordered-subsets expectation maximization (2D-OSEM) (16 subsets and 4 iterations) or (2) threedimensional OSEM, maximum a posteriori (MAP) estimation (FMAP) (two 3D-OSEM and 20 MAP iterations), having a voxel size of $0.5 \times$ $0.5 \times 0.8$ and $0.4 \times 0.4 \times 0.8 \mathrm{~mm}^{3}$, respectively.

Image analysis. PET image reconstruction was performed using ASIPro VM (CTI Concorde Microsystem Analysis Tools and System Setup/Diagnostics Tool). Image analysis and quantification of radioactivity uptake in volumes of interest (VOIs) were performed using BrainVisa/Anatomist version 3.1 (Cointepas et al., 2001). Spinal cord delineation was made by manual segmentation on the summed-frame image spanning the last $30 \mathrm{~min}$ of the PET acquisition. Specifically, a region of interest (ROI) was created on each transaxial PET section by drawing a $3 \times 3$-voxel cross (total volume of 1 or $0.64 \mathrm{~mm}^{3}$ for $2 \mathrm{D}$ OSEM or FMAP reconstruction, respectively), centered on the spinal cord, and perpendicular to the spinal cord axis. Adjacent ROIs were grouped (for measurement) over a length of $\sim 3 \mathrm{~cm}$, yielding a longitudinal 3D representation of the spinal cord. Radioactivity uptake values were quantified in becquerels per cubic millimeter of tissue and converted into $\% \mathrm{ID} / \mathrm{cc}$, corrected for fluorine-18 decay.

In a subset of the studies $(n=12)$, the $\left[{ }^{18} \mathrm{~F}\right] \mathrm{DPA}-714$ scan was immediately followed by a second $\left[{ }^{18} \mathrm{~F}\right]$ fluoride PET acquisition. Fluoride anions exhibit high affinity to bone tissue and outline the vertebrae of the spinal column. Therefore, the anatomical accuracy of the spinal cord ROIs drawn on the $\left[{ }^{18} \mathrm{~F}\right] \mathrm{DPA}-714$ PET images was qualitatively validated by superimposing the corresponding $\left[{ }^{18} \mathrm{~F}\right]$ fluoride PET image.

Statistical analysis. Statistical analysis was made using GraphPad Prism 4 Software. Unless otherwise stated, all data are expressed as mean \pm SEM. Comparison of TSPO expression in spinal cords of EAE and control rats, as indicated by Western blot analysis, was made using one-tailed Student's $t$ test. In biodistribution and imaging studies, comparisons of $\left[{ }^{18} \mathrm{~F}\right] \mathrm{DPA}-714$ uptake in spinal cords of each group were made using one-way ANOVA, followed by Bonferroni's post hoc test. Specifically, $\left[{ }^{18} \mathrm{~F}\right] \mathrm{DPA}-714$-injected rats were assigned into any of the four following groups: (1) control (CFA-injected) rats, (2) EAE-induced rats without challenge, (3) EAE-induced rats with $(R, S)$-PK11195 challenge, or (4) EAE-induced rats challenged with DPA-714. Bonferroni's post hoc test analysis was used to compare $\left[{ }^{18} \mathrm{~F}\right] \mathrm{DPA}-714$ uptake in spinal cords of each group with those of EAE-induced rats (group 2). The level of significance was regularly set at $p<0.05$. 

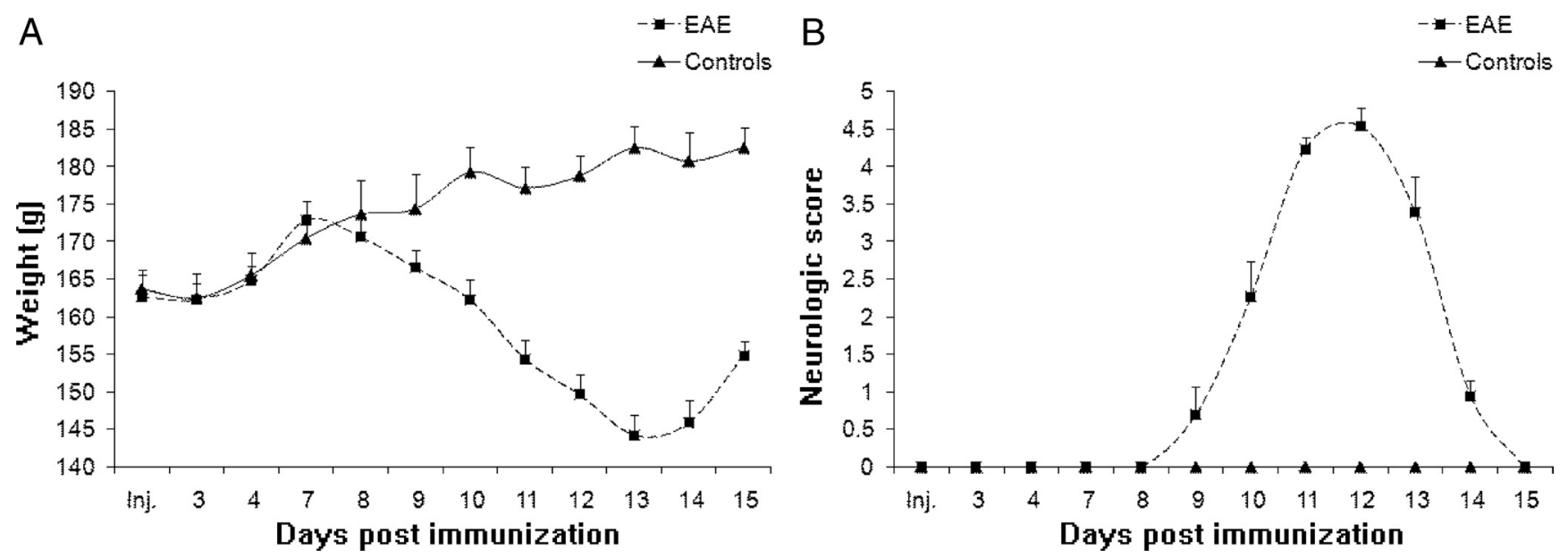

Figure 1. A representative EAE course in female Lewis rats after immunization with gpMBP ${ }_{68-86}$ fragment. Injection of gpMBP $68-86$ emulsion (25 $\left.\mu \mathrm{g} / \mathrm{rat}_{\mathrm{r}} n=13\right)$ induces weight loss $(\boldsymbol{A})$, succeeded by a monophasic acute illness $(\boldsymbol{B})$ that peaks on $11-12 \mathrm{dpi}$, and regresses completely on $\sim 15 \mathrm{dpi}$. No clinical symptoms or weight loss are observed in control, CFA-injected rats ( $n=$ 7). Neurological scoring: 0 , no symptoms; 1 , flaccid tail; 2 , hindlimb weakness; 3 , paraparesis; 3.5 , unilateral hindlimb paralysis; 4 , bilateral hindlimb paralysis; and 5, bilateral hindlimb paralysis and incontinence. Results are expressed as mean + SEM.

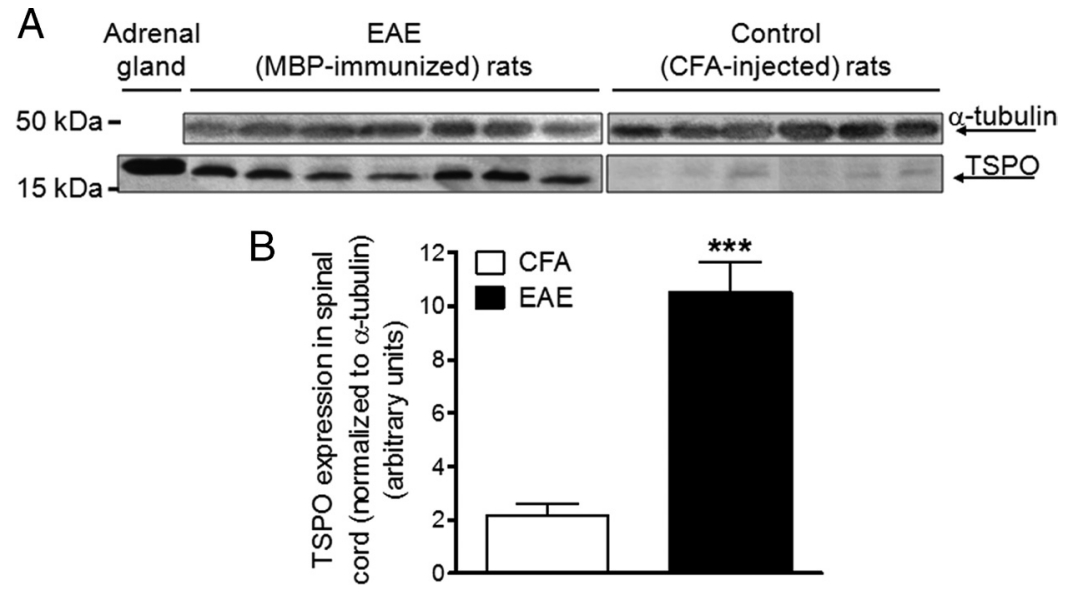

Figure 2. Increased TSPO expression in spinal cords of EAE-induced rats compared with those of controls. Spinal cord homogenates were prepared from EAE rats $(n=7)$ at maximum deficit (neurologic scores of $4-5)$ and controls $(n=6)$ and analyzed for their TSPO content by Western blot. Higher levels of TSPO are present in spinal cords of EAE-induced rats versus those of controls (A). First lane on the left, an adrenal gland extract, used as positive control for TSPO expression. TSPO levels (normalized to those of $\alpha$-tubulin) are approximately fivefold higher in spinal cords of EAE rats $(\boldsymbol{B}) .{ }^{* * *} p=0.0001$.

\section{Results}

Clinical course of gpMBP $68-86$-induced EAE in female Lewis rats

Figure 1 illustrates a typical clinical course of EAE in female Lewis rats after immunization with gpMBP ${ }_{68-86}$ peptide $(25 \mu \mathrm{g} / \mathrm{rat})$ versus control, CFA-injected rats. In response to the antigenic challenge, the rats developed an acute monophasic disease with an incidence of $100 \%$. Clinically, the acute phase of gradually ascending hindlimb paralysis was preceded by significant weight loss at 7-8 d post-immunization (dpi). The onset of neurological deficits occurred at $\sim 9 \mathrm{dpi}$, reaching a disease peak score of $4.5 \pm 0.9$ at $11.4 \pm 0.2 \mathrm{dpi}$. This acute clinical disease had resolved completely by $\sim 15 \mathrm{dpi}$, with no additional relapses. No weight loss or apparent clinical symptoms occurred within CFAinjected rats (Fig. 1). Accordingly, all in vivo studies were performed on 10-12 dpi using EAE rats with neurological scores of 4-5 and matched CFA-injected controls.
Increased TSPO expression in spinal cords of EAE rats versus controls

The extent of TSPO expression in spinal cords of EAE-induced rats $(n=7)$ versus those of controls $(n=6)$ was quantified by Western blot analysis. As presented in Figure 2, TSPO levels in spinal cords of EAEinduced rats were approximately fivefold higher than those of control animals. Furthermore, immunohistochemical staining of spinal cord tissue from EAE $(n=5)$ and control $(n=2)$ rats confirmed the enhanced expression of TSPO in spinal cords of EAE rats (Fig. 3), identifying activated microglia/macrophages, rather than reactive astrocytes, as the cellular source of TSPO expression.

Enhanced specific uptake of $\left[{ }^{18} \mathrm{~F}\right] \mathrm{DPA}$ 714 in spinal cords of EAE-induced rats: biodistribution studies

The distribution of radioactivity was measured at $1 \mathrm{~h}$ after intravenous injection of $\left[{ }^{18} \mathrm{~F}\right] \mathrm{DPA}-714$ into $\operatorname{EAE}(n=15)$ and control $(n=16)$ rats. As shown in Figure 4 , radioactivity uptake values in the thoracic and lumbar spinal cords of EAE-induced rats were fourfold to fivefold higher, respectively, than those of control animals. Importantly, the enhanced uptake of $\left[{ }^{18} \mathrm{~F}\right] \mathrm{DPA}-$ 714 in spinal cords of EAE-induced rats was confirmed to be TSPO specific, because competition with an excess of unlabeled $(R, S)$-PK11195 or DPA-714 significantly reduced \%ID/g values in spinal cords of EAE rats (Fig. 4). The extent of reduction in $\left[{ }^{18} \mathrm{~F}\right] \mathrm{DPA}-714$ uptake in spinal cord ranged from 42 to $55 \%$, depending on the region of the cord and on the unlabeled competitor used. Contrary to the different levels of $\left[{ }^{18} \mathrm{~F}\right] \mathrm{DPA}-714$ in spinal cords of EAE rats and controls, radioactivity concentrations in blood samples from both groups were on par. That, and the fact that rats were perfused with PBS before collection of tissues, rules out that differences in CNS blood flow and/or permeability between EAE and control rats could have affected the measured uptake of $\left[{ }^{18} \mathrm{~F}\right] \mathrm{DPA}-714$ in spinal cords. In challenge 


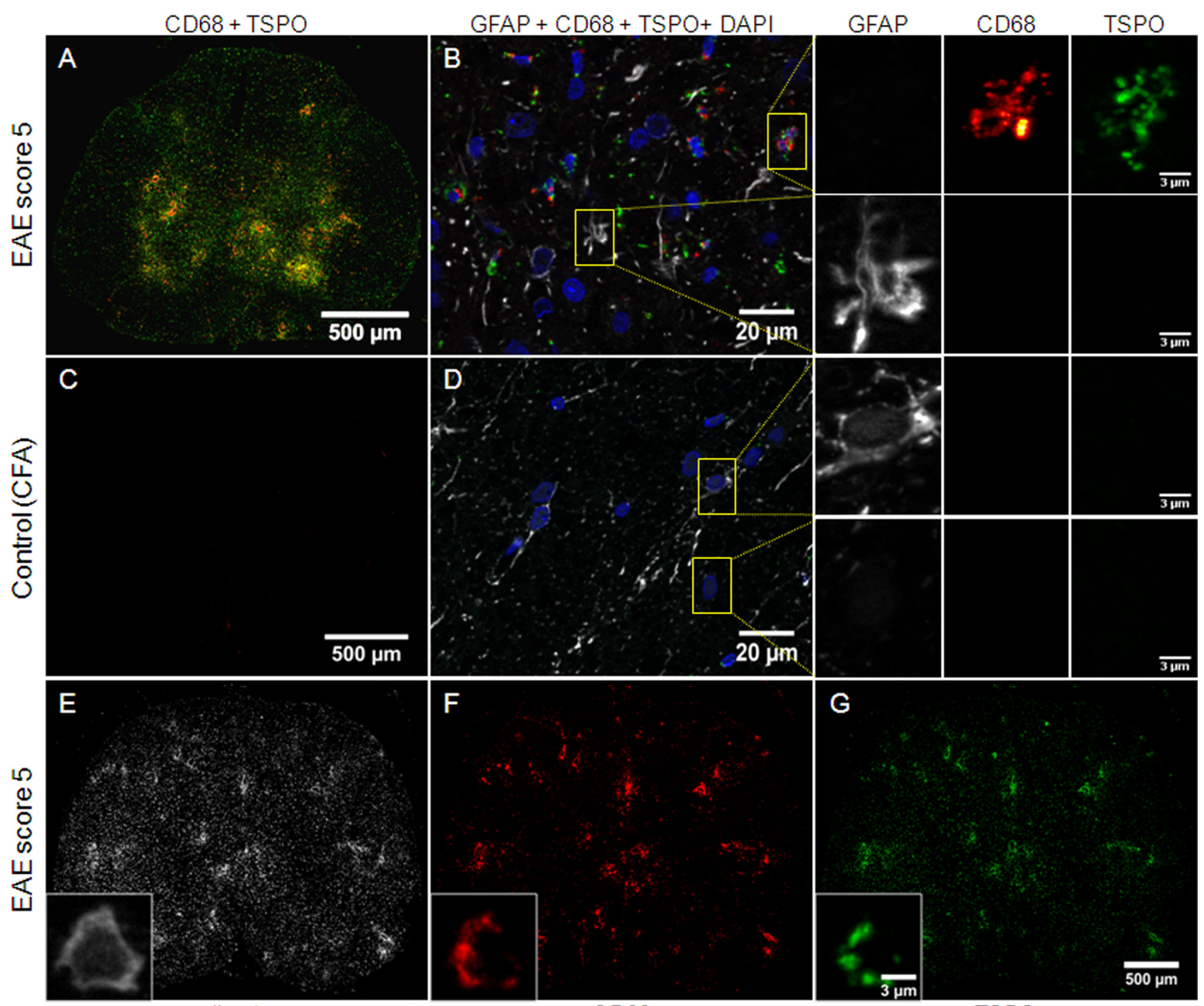

lba-1

CD68

TSPO

Figure 3. TSPO expression in spinal cords of EAE-induced rats is associated with activated microglia/macrophages. Paraffin-embedded spinal cord tissue sections ( $5 \mu \mathrm{m})$ were stained, as described in Materials and Methods, for nucleus (blue DAPI), for activated microglia/macrophages [red CD68 $(\boldsymbol{A}-\boldsymbol{D}, \boldsymbol{F})$ or lba-1 (E)], reactive astrocytes [white GFAP $(\boldsymbol{B}, \boldsymbol{D})$ ], and TSP0 expression [green NP155 $(\boldsymbol{A}-\boldsymbol{D}, \boldsymbol{G})]$. Representative immunohistochemical staining of spinal cord tissues from $E A E(\boldsymbol{A}, \boldsymbol{B}, \boldsymbol{E}-\boldsymbol{G})$ and control $(\boldsymbol{C}, \boldsymbol{D})$ rats identify activated microglia/macrophages, rather than reactive astrocytes, as the cellular source of TSPO expression.

studies using an excess of unlabeled $(R, S)-\mathrm{PK} 11195$ or DPA-714, 2.5-fold and 5-fold higher levels of radioactivity were measured in blood, respectively (Fig. 4). This rise in radioactivity concentration in blood is explained, among others, by displacement of $\left[{ }^{18} \mathrm{~F}\right] \mathrm{DPA}-714$ from TSPO binding sites in peripheral organs by unlabeled $(R, S)$-PK11195 or DPA-714.

\section{Enhanced specific uptake of $\left[{ }^{18} \mathrm{~F}\right] \mathrm{DPA}-714$ in spinal cords of} EAE-induced rats: $P E T$ imaging

The ability to detect and quantify neuroinflammatory lesions in spinal cords of rats in vivo was evaluated by microPET imaging using EAE-induced and CFA-injected control rats. Biodistribution data indicated that $\left[{ }^{18} \mathrm{~F}\right] \mathrm{DPA}-714$ uptake in the spinal cord was significantly lower than in other TSPO-rich peripheral organs (data not shown). Particularly, the range of $\left[{ }^{18} \mathrm{~F}\right] \mathrm{DPA}-714$ uptake values in spinal cords was $0.10-0.22$ and $0.43-1.22 \% \mathrm{ID} / \mathrm{g}$ for control and EAE-induced rats, respectively. Thus, to improve count statistics of $\left[{ }^{18} \mathrm{~F}\right] \mathrm{DPA}-714$ in spinal cords, a static image was generated for each scan by summing the last 30 min of the acquisition. This timeframe was selected based on our previous reports, indicating that, at $30 \mathrm{~min}$ after intravenous injection of $\left[{ }^{18} \mathrm{~F}\right] \mathrm{DPA}-714$, uptake of the radiotracer in the CNS and in TSPO-rich organs reached a plateau (Chauveau et al., 2009; Martín et al., 2010). To account for the small diameter of the rat spinal cord, ranging from 1 to $3 \mathrm{~mm}$ (Behr et al., 2004), PET images were reconstructed using both conventional 2D-OSEM (Fig. 5A,B) and FMAP (Fig. 5C,D) reconstruction algorithms (detailed in Materials and Methods). Delineation of the spinal cord was made along the transaxial (longitudinal) axis by drawing on each transverse PET section a $3 \times 3$-voxel cross, centered on the spinal cord (Fig. $5 E$ ), thereby yielding a longitudinal $3 \mathrm{D}$ representation of the spinal cord (Fig. $5 F$ ).

Characteristic images of $\left[{ }^{18} \mathrm{~F}\right] \mathrm{DPA}-714$ in control and EAE rats shown in Figure 6 illustrate the increased radioactivity uptake in spinal cords of EAE-induced rats. Quantification of [ $\left.{ }^{18} \mathrm{~F}\right] \mathrm{DPA}-$ 714 uptake using FMAP-reconstructed images revealed 2.6-fold 


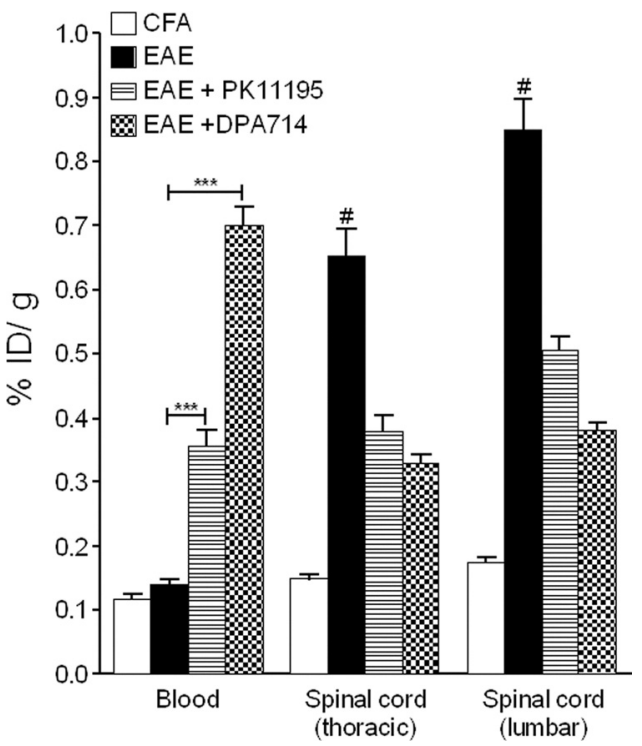

Figure 4. Enhanced specific uptake of $\left[{ }^{18} \mathrm{~F}\right] \mathrm{DPA}-714$ in spinal cords of EAE-induced rats versus those of controls in biodistribution studies. Distribution of radioactivity was measured at $1 \mathrm{~h}$ after intravenous injection of $\left[{ }^{18} \mathrm{~F}\right] \mathrm{DPA}-714$ into control $(n=16)$ or EAE-induced $(n=15)$ rats, revealing fourfold to fivefold higher radioactivity uptake values in spinal cords of EAE rats. In competition studies, an excess of unlabeled (R,S)-PK11195 $(n=8)$ or DPA-714 $(n=7)$ was injected into EAE rats 30 min after injection of $\left[{ }^{18} \mathrm{~F}\right] \mathrm{DPA}-714$. Radioactivity uptake in spinal cords of EAE rats was reduced by $\sim 50 \%$ after injection of either TSPO ligand, signifying specific binding of the tracer. ${ }^{* * *} p<0.001$; ${ }^{\#} p<0.001$, highly statistically significant difference with respect to the remaining three groups.
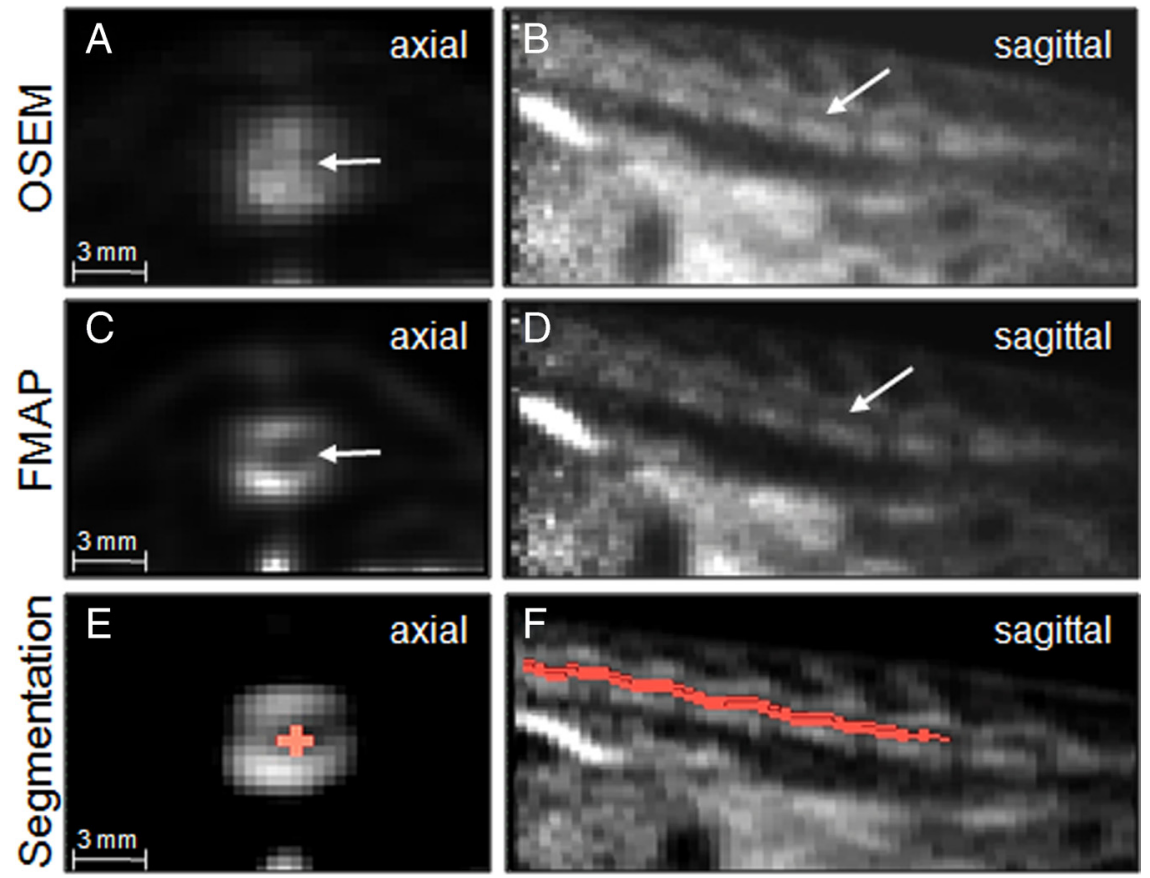

Figure 5. PET image reconstruction and segmentation of the spinal cord region after injection of $\left[{ }^{18} \mathrm{~F}\right] \mathrm{DPA}-714$. Radioactivity concentrations in the spinal cords were measured using 30-min (summed-frame) images that correspond to the last $30 \mathrm{~min}$ of each dynamic acquisition. A representative PET image of an EAE-induced rat is demonstrated, reconstructed using both 2D-OSEM $(\boldsymbol{A}, \boldsymbol{B})$ and 3D-0SEMMAP (FMAP) $(\boldsymbol{C}, \boldsymbol{D})$ reconstruction algorithms. Spinal cord regions (indicated by arrows) were segmented by drawing a $3 \times 3$-voxel cross per plane (centered on the cord), along the axial axis $(\boldsymbol{E})$, yielding an approximated 3D representation of the spinal cord $(\boldsymbol{F})$.

higher levels in spinal cords of EAE-induced rats versus controls (Fig. 6E, Table 1). Using both 2D-OSEM and FMAP reconstruction algorithms, a statistically significant reduction in $\left[{ }^{18} \mathrm{~F}\right] \mathrm{DPA}-$ 714 uptake was measured in spinal cords of EAE-induced rats after displacement with an excess of unlabeled $(R, S)$-PK11195 (28-39\% reduction) or DPA-714 (43-50\% reduction) (Table 1). Taking the biodistribution results as a reference (Fig. 3 ), the calculated in vivo uptake values in spinal cords were closer using FMAP reconstruction rather than 2D-OSEM, suggesting that FMAP reconstruction had reduced partial volume effect artifacts.

Delineation of spinal cord regions on $\left[{ }^{18} \mathrm{~F}\right] \mathrm{DPA}-714$ PET images was made possible by the well-defined gradient between the surrounding vertebrae and the confined spinal cord (Figs. 5, 6). To confirm spinal cord delineation, in a subset of the imaging studies $(n=12)$, the $\left[{ }^{18} \mathrm{~F}\right] \mathrm{DPA}-714$ PET acquisition was followed by a second PET scan, after injection of $\left[{ }^{18} \mathrm{~F}\right]$ fluoride, a bone tracer that provides precise visualization of the spinal column (data not shown). The accuracy of spinal cord delineation on the $\left[{ }^{18} \mathrm{~F}\right] \mathrm{DPA}-714$ PET images was evaluated on each transaxial PET section, using the $\left[{ }^{18} \mathrm{~F}\right]$ fluoride image corresponding to each individual animal. There was an overall overlap of $90 \pm 3 \%$ between the delineated spinal cord on $\left[{ }^{18} \mathrm{~F}\right] \mathrm{DPA}-714$ images and the orientation of the cord on $\left[{ }^{18} \mathrm{~F}\right]$ fluoride images.

\section{Discussion}

MS is a multifocal disease of the CNS, characterized by inflammation, demyelination, and axonal loss. Activated microglia/ macrophages play a central role in the inflammatory process of autoimmune demyelinating diseases and particularly in the immunopathogenesis of MS and EAE (Raivich and Banati, 2004; Sanders and De Keyser, 2007). Consequently, many studies have addressed the inflammatory component of this complex disease.

We demonstrate here that the TSPO ligand $\left[{ }^{18} \mathrm{~F}\right] \mathrm{DPA}-714$ is a specific marker for imaging microglial activation in the spinal cord of EAE rats by PET, offering a quantitative and sensitive insight, at the molecular level, into neuroinflammatory processes.

\section{Detecting neuroinflammation in EAE using $\left[{ }^{18}\right.$ F $]$ DPA-714}

EAE is the collective name for different animal models of MS. EAE models reproduce hallmarks of the disease and, similarly to MS, are heterogeneous in their clinical, neuropathological, and immunological aspects (Gold et al., 2006). The MBP-induced EAE model was chosen here for imaging microglial activation using $\left[{ }^{18} \mathrm{~F}\right] \mathrm{DPA}-714$ and PET for several reasons. The $\mathrm{MBP}_{68-86}$ fragment is the dominant encephalitogenic MBP epitope for Lewis rats. Immunization with MBP elicits an acute monophasic illness, with $100 \%$ incidence, and over a reproducible clinical course. Interestingly, this immunization protocol is characterized by mononuclear inflammatory lesions in the spinal cord, rarely affecting the brain (Stepaniak et al., 1995; Duplan et al., 2003). Moreover, this acute spinal cord inflammation is characterized by considerable activation of microglia/macrophages, which is coupled to an enhanced mitochondrial expression of TSPO (Banati, 2002). Using the same animal model, Mattner et al. (2005) have shown 

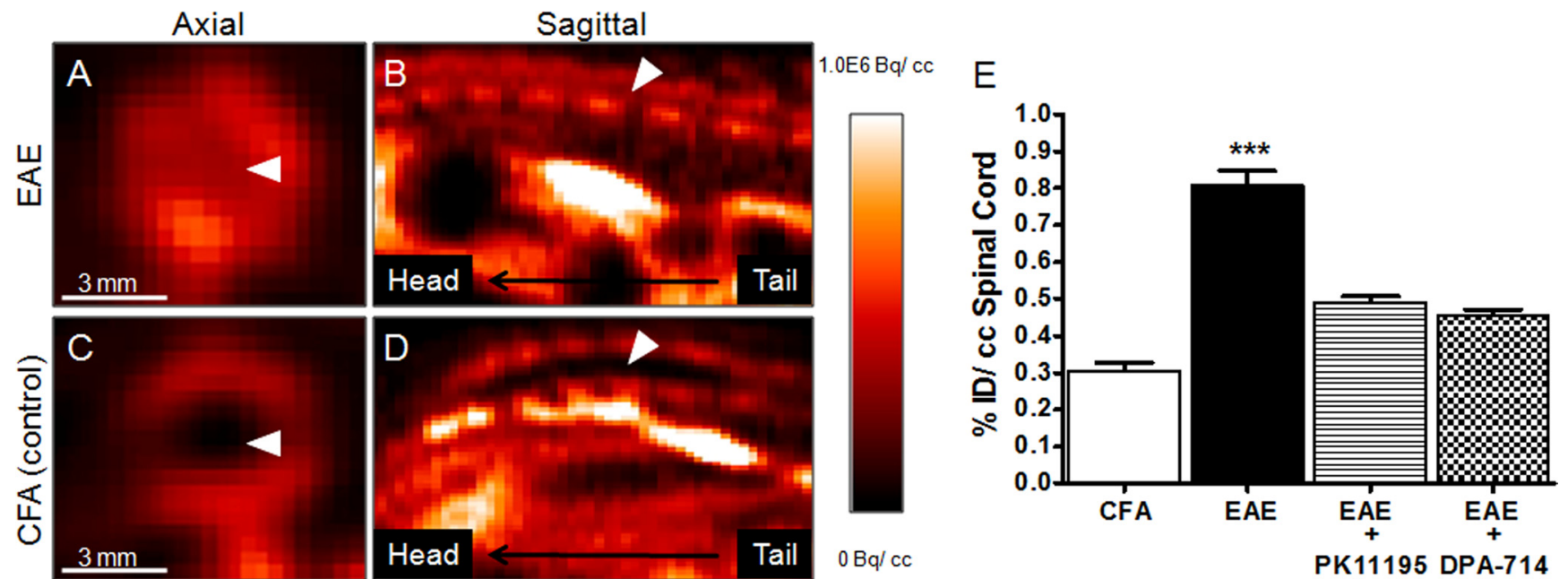

Figure 6. Enhanced uptake of $\left[{ }^{18} \mathrm{~F}\right] \mathrm{DPA}-714$ in spinal cords of EAE-induced rats versus controls using microPET. Representative images of increased uptake of [ $\left.{ }^{18} \mathrm{~F}\right] \mathrm{DPA}-714$ in spinal cords of EAE-induced rats $(\boldsymbol{A}, \boldsymbol{B})$ compared with CFA-injected controls $(\boldsymbol{C}, \boldsymbol{D})$ (spinal cords are indicated by arrowheads). Using FMAP image reconstruction algorithm, radioactivity concentration in spinal cords of EAE-induced rats $(n=8)$ was 2.6-fold higher than that of controls $(n=8)(E)$. Displacement of $\left[{ }^{18} \mathrm{~F}\right] \mathrm{DPA}$-714 binding in spinal cord after administration of either unlabeled $(R, S)$-PK11195 $(n=8)$ or DPA-714 $(n=7)(1 \mathrm{mg} / \mathrm{kg})$ could be demonstrated and quantified by PET. ${ }^{* *} p<0.001$, compared with the other three groups.

Table 1. Comparison of $\left[{ }^{18} \mathrm{~F}\right] \mathrm{DPA}-714$ uptake in spinal cords of control and EAE-induced rats as obtained using 2D-0SEM and FMAP reconstruction algorithms

\begin{tabular}{lllll}
\hline & CFA $(n=8)$ & EAE $(n=8)$ & $\begin{array}{l}\text { EAE }+ \text { PK11195 } \\
(n=8)\end{array}$ & $\begin{array}{l}\text { EAE }+ \text { DPA-714 } \\
(n=7)\end{array}$ \\
\hline 2D-0SEM & $0.42 \pm 0.03$ & $0.88 \pm 0.03^{*}$ & $0.63 \pm 0.02$ & $0.43 \pm 0.01$ \\
FMAP & $0.31 \pm 0.02$ & $0.81 \pm 0.04^{*}$ & $0.49 \pm 0.01$ & $0.45 \pm 0.02$
\end{tabular}

Radioactivity uptake values in spinal cords were calculated from PET images using both 2D-0SEM and FMAP reconstruction algorithms. Challenge studies were performed by injecting unlabeled (R,S)-PK11195 or DPA-714 in excess, 30 min after injection of the radioligand. Results are expressed as mean \pm SEM \% ID/cc and represent the last $30 \mathrm{~min}$ (or 20 min in challenge studies) of a 68 min dynamic scan. Sample size $(n)$ is indicated in brackets. ${ }^{*} p<0.001$, highly statistically significant difference with respect to the remaining three columns.

that the degree of inflammation increases from rostral to caudal parts of the spinal cord and that the extent of lumbar/sacral inflammation was in good agreement with the clinical score of the rats.

Western blot analysis confirmed a fivefold higher expression of TSPO in spinal cords of EAE rats with respect to controls, suggesting that, using $\left[{ }^{18} \mathrm{~F}\right] \mathrm{DPA}-714$, it could be possible to detect differences between the two groups. Additionally, IHC staining (Fig. 3) illustrated that the TSPO expression was colocalized with microglia/macrophages (CD68/Iba-1) rather than with astrocytes (GFAP), as has been demonstrated for this model (Mattner et al., 2005). Notably, previous reports have indicated that no radiolabeled metabolites were detected in the CNS of rats up to $60 \mathrm{~min}$ after injection of $\left[{ }^{18} \mathrm{~F}\right] \mathrm{DPA}-714$ (Chauveau et al., 2009), sustaining that potential differences in radioactivity concentrations measured in spinal cords would be entirely attributed to the intact radiotracer. In concordance with the Western blot, biodistribution studies revealed fourfold to fivefold higher levels of $\left[{ }^{18} \mathrm{~F}\right] \mathrm{DPA}-714$ in spinal cords of EAE rats versus controls. Consistent with the reports of increased inflammation in the caudal parts of the spinal cord, higher uptake of $\left[{ }^{18} \mathrm{~F}\right] \mathrm{DPA}-714$ was measured in the lumbar part compared with the thoracic part of the cord. Injecting an excess of unlabeled $(R, S)$-PK11195 or DPA-714, 30 min after $\left[{ }^{18} \mathrm{~F}\right] \mathrm{DPA}-714$, reduced spinal cord uptake in EAE rats to the level of uptake in control animals (Fig. 4).

\section{PET methodology}

The diameter of rat spinal cord is the same as the resolution limit of the microPET scanner ( $1.35 \mathrm{~mm}$ FWHM). Consequently,
PET imaging of the rat spinal cord is a challenge, and specific methods were designed to obtain the best possible visualization of spinal cords. First, the rostral lumbar and caudal thoracic parts of the cord were positioned at the center of the PET FOV, in which spatial resolution is highest. Second, to improve count statistics, radioactivity counts were collected over the last $30 \mathrm{~min}$ of each scan. Third, the FMAP reconstruction algorithm, although more time consuming than classical 2D-OSEM, was used to improve resolution. The results (Fig. 5, Table 1) indicate that differences in uptake of $\left[{ }^{18} \mathrm{~F}\right] \mathrm{DPA}-714$ in spinal cords could be detected and quantified using both reconstruction algorithms. FMAP reconstruction better reflected the reference (biodistribution) results, corroborating its value in reducing errors attributable to partial volume effects. Complete concordance between biodistribution and PET data, vis-à-vis $\left[{ }^{18} \mathrm{~F}\right] \mathrm{DPA}-714$ uptake in spinal cords, was not expected in these studies for several reasons: (1) delineation of the cords reflected neither their precise anatomy nor their entire length because of FOV limitations; and (2) definition of the spinal cord region in $\left[{ }^{18} \mathrm{~F}\right] \mathrm{DPA}-714$ images was based on radioactivity uptake in the vertebrate, and enhanced precision should be expected using microPET-computed tomography scanners.

\section{Pivotal role of activated microglia/macrophages in neuroinflammation}

Activated microglia/macrophages are key effectors in a variety of immune-associated neuropathies and disorders (Minagar et al., 2002; Nelson et al., 2002; Rock et al., 2004). Typically, microglia are rapidly activated in response to subtle changes in their environment, proliferate, and migrate to remote projection sites along the injured neuronal path. The principal argument for imaging microglial activation in various CNS inflammatory conditions lies in their involvement in neuroinflammatory processes, rendering them a generic marker of many ongoing CNS pathologies.

Microglial activation has been demonstrated in early and late active MS lesions and in inactive ones (Brück et al., 1995; Stadelmann and Brück, 2008). Notably also, microglial activation has been documented in the NAWM and in cortical gray matter lesions, mostly in chronic progressive forms of MS. Such relatively 
small and diffuse pathological changes present a challenge vis-àvis their detection by in vivo imaging (Kutzelnigg et al., 2007; Zeis et al., 2008).

\section{Monitoring neuroinflammation using MRI and PET}

Imaging neuroinflammation has been reported using both MRI and PET (Floris et al., 2004; Vellinga et al., 2008). Monitoring Gd-enhancing lesions with MRI, for example, has been generally recognized as a reliable means for tracking changes associated with inflammatory pathologies in MS. Nonetheless, Gd enhancement is not specific for active demyelinating lesions, represents $\mathrm{BBB}$ leakage rather than true cellular invasion into the CNS, and is expected to be less sensitive in detecting pathological processes trapped behind a closed/repaired BBB (Floris et al., 2004; Metz et al., 2007).

MRI using ultra-small superparamagnetic iron-oxide (USPIO) nanoparticles has been successfully used in MS patients and EAE models for imaging neuroinflammation as a result of CNS infiltration of peripheral macrophages that have phagocytosed the USPIO particles. However, its sensitivity appears to be most compatible for imaging relatively large waves of peripheral macrophage infiltration rather than diffused and inconspicuous pathological changes, as in the NAWM of numerous MS patients (Dousset et al., 2006; Vellinga et al., 2008). Thus, there is need for complementary imaging approaches with better molecular sensitivity, such as PET.

Imaging neuroinflammation by PET has been primarily demonstrated using radiolabeled ligands that target the TSPO (Cagnin et al., 2007; Radu et al., 2007; Winkeler et al., 2010). Because TSPO expression in the healthy CNS is low, increased CNS uptake of TSPO-targeted radioligands is unequivocally indicative of neuroinflammatory processes (Anholt et al., 1985; Mattner et al., 2008). Pertaining to imaging neuroinflammation is the fact that TSPO expression in microglia occurs early in their activation and persists throughout their different stages of activation, rendering the TSPO an early and persistent marker of neuroinflammation (Kreutzberg, 1996; Banati, 2002). Hence, imaging neuroinflammation by PET using TSPO-specific ligands should provide increased sensitivity, because it depicts a cellular activity that does not rely solely on invasion of peripheral macrophages or on BBB integrity.

Accordingly, this approach has been applied to different CNS inflammatory pathologies and conditions in human patients and animal models. Using $\left[{ }^{11} \mathrm{C}\right] \mathrm{PK} 11195$, increased binding in MS lesions has been demonstrated by postmortem data and in vivo PET (Banati et al., 2000, 2002). However, shortcomings of $\left[{ }^{11} \mathrm{C}\right] \mathrm{PK} 11195$ as a PET ligand result in underestimation of TSPO expression and a reduced sensitivity (Banati, 2002; Cagnin et al., 2007). Previous publications (Chauveau et al., 2009; Martín et al., 2010) pointed at $\left[{ }^{18} \mathrm{~F}\right] \mathrm{DPA}-714$ as a potentially superior TSPO ligand for imaging activated microglia by PET, prompting its investigation in additional animal models of neuroinflammation.

The presented results indicate that neuroinflammation in spinal cords of EAE-induced rats can be detected using $\left[{ }^{18} \mathrm{~F}\right] \mathrm{DPA}-$ 714 and PET. Imaging of rat spinal cord in vivo poses particular challenge because of its small diameter (1-3 mm) (Behr et al., 2004), and spinal cord MRI is rarely performed in experimental studies. Still, much of the physical disability related to MS is probably mediated by spinal cord lesions, which can progress independently of brain disease. Therefore, improvements in spinal cord imaging techniques are of considerable interest. Their value has been demonstrated in establishing an early diagnosis in MS and in improving the correlation between clinical and MRI- based classification (Bot et al., 2004; Agosta et al., 2007; Ciccarelli et al., 2007). Previous reports have highlighted the potential role of spinal cord lesions in predicting the evolution of disability in MS (Agosta et al., 2007), asserting the utility of in vivo spinal cord imaging for promoting MS research, monitoring, and, hopefully, therapy.

\section{Summary and conclusions}

We report here for the first time, imaging a central component of neuroinflammation, the activation of microglia, in spinal cords of EAE-induced rats using a TSPO-specific PET radioligand, $\left[{ }^{18}\right.$ F]DPA-714. Such studies should provide more insight into microglial activation in different forms of MS, as well as in other neuroinflammatory (spinal cord) diseases and conditions, and ultimately lay a foundation for improving clinical diagnosis and treatment of MS patients.

\section{References}

Agosta F, Absinta M, Sormani MP, Ghezzi A, Bertolotto A, Montanari E, Comi G, Filippi M (2007) In vivo assessment of cervical cord damage in MS patients: a longitudinal diffusion tensor MRI study. Brain 130:2211-2219.

Anholt RR, De Souza EB, Oster-Granite ML, Snyder SH (1985) Peripheraltype benzodiazepine receptors: autoradiographic localization in wholebody sections of neonatal rats. J Pharmacol Exp Ther 233:517-526.

Banati RB (2002) Visualising microglial activation in vivo. Glia 40:206-217.

Banati RB (2003) Neuropathological imaging: in vivo detection of glial activation as a measure of disease and adaptive change in the brain. Br Med Bull 65:121-131.

Banati RB, Newcombe J, Gunn RN, Cagnin A, Turkheimer F, Heppner F, Price G, Wegner F, Giovannoni G, Miller DH, Perkin GD, Smith T, Hewson AK, Bydder G, Kreutzberg GW, Jones T, Cuzner ML, Myers R (2000) The peripheral benzodiazepine binding site in the brain in multiple sclerosis: quantitative in vivo imaging of microglia as a measure of disease activity. Brain 123:2321-2337.

Barkhof F, Calabresi PA, Miller DH, Reingold SC (2009) Imaging outcomes for neuroprotection and repair in multiple sclerosis trials. Nat Rev Neurol 5:256-266.

Behr VC, Weber T, Neuberger T, Vroemen M, Weidner N, Bogdahn U, Haase A, Jakob PM, Faber C (2004) High-resolution MR imaging of the rat spinal cord in vivo in a wide-bore magnet at 17.6 Tesla. MAGMA 17:353-358.

Bot JC, Barkhof F, Polman CH, Lycklama à Nijeholt GJ, de Groot V, Bergers E, Ader HJ, Castelijns JA (2004) Spinal cord abnormalities in recently diagnosed MS patients: added value of spinal MRI examination. Neurology 62:226-233.

Bradford MM (1976) A rapid and sensitive method for the quantitation of microgram quantities of protein utilizing the principle of protein-dye binding. Anal Biochem 72:248-254.

Brück W, Porada P, Poser S, Rieckmann P, Hanefeld F, Kretzschmar HA, Lassmann H (1995) Monocyte/macrophage differentiation in early multiple sclerosis lesions. Ann Neurol 38:788-796.

Cagnin A, Kassiou M, Meikle SR, Banati RB (2007) Positron emission tomography imaging of neuroinflammation. Neurotherapeutics 4:443-452.

Chauveau F, Van Camp N, Dollé F, Kuhnast B, Hinnen F, Damont A, Boutin H, James M, Kassiou M, Tavitian B (2009) Comparative evaluation of the translocator protein radioligands 11C-DPA-713, 18F-DPA-714, and $11 \mathrm{C}-\mathrm{PK} 11195$ in a rat model of acute neuroinflammation. J Nucl Med 50:468-476.

Ciccarelli O, Wheeler-Kingshott CA, McLean MA, Cercignani M, Wimpey K, Miller DH, Thompson AJ (2007) Spinal cord spectroscopy and diffusion-based tractography to assess acute disability in multiple sclerosis. Brain 130:2220-2231.

Cleveland DW, Fischer SG, Kirschner MW, Laemmli UK (1977) Peptide mapping by limited proteolysis in sodium dodecyl sulfate and analysis by gel electrophoresis. J Biol Chem 252:1102-1106.

Cointepas Y, Mangin J-F, Garnero L, Poline J-B, Benali H (2001) BrainVISA: software platform for visualization and analysis of multi-modality brain data. Neuroimage 13:S98. 
Damont A, Hinnen F, Kuhnast B, Schöllhorn-Peyronneau M, James M, Luus C, Tavitian B, Kassiou M, Dollé F (2008) Radiosynthesis of [18F]DPA714 , a selective radioligand for imaging the translocator protein $(18 \mathrm{kDa})$ with PET. J Labelled Comp Radiopharm 51:286-292.

Dollé F, Luus C, Reynolds A, Kassiou M (2009) Radiolabelled molecules for imaging the translocator protein $(18 \mathrm{kDa})$ using positron emission tomography. Curr Med Chem 16:2899-2923.

Dousset V, Brochet B, Deloire MS, Lagoarde L, Barroso B, Caille JM, Petry KG (2006) MR imaging of relapsing multiple sclerosis patients using ultrasmall-particle iron oxide and compared with gadolinium. AJNR Am J Neuroradiol 27:1000-1005.

Dubois-Dalcq M, Williams A, Stadelmann C, Stankoff B, Zalc B, Lubetzki C (2008) From fish to man: understanding endogenous remyelination in central nervous system demyelinating diseases. Brain 131:1686-1700.

Duplan V, Dutartre P, Mars LT, Liblau RS, Druet P, Saoudi A (2003) LF 15-0195 inhibits the development of rat central nervous system autoimmunity by inducing long-lasting tolerance in autoreactive CD4 T cells. J Immunol 170:2179-2185.

Floris S, Blezer EL, Schreibelt G, Döpp E, van der Pol SM, SchadeeEestermans IL, Nicolay K, Dijkstra CD, de Vries HE (2004) Blood-brain barrier permeability and monocyte infiltration in experimental allergic encephalomyelitis: a quantitative MRI study. Brain 127:616-627.

Gold R, Linington C, Lassmann H (2006) Understanding pathogenesis and therapy of multiple sclerosis via animal models: 70 years of merits and culprits in experimental autoimmune encephalomyelitis research. Brain 129:1953-1971.

James ML, Fulton RR, Vercoullie J, Henderson DJ, Garreau L, Chalon S, Dolle F, Costa B, Selleri S, Guilloteau D, Kassiou M (2008) DPA-714, a new translocator protein-specific ligand: synthesis, radiofluorination, and pharmacologic characterization. J Nucl Med 49:814-822.

Ji B, Maeda J, Sawada M, Ono M, Okauchi T, Inaji M, Zhang MR, Suzuki K, Ando K, Staufenbiel M, Trojanowski JQ, Lee VM, Higuchi M, Suhara T (2008) Imaging of peripheral benzodiazepine receptor expression as biomarkers of detrimental versus beneficial glial responses in mouse models of Alzheimer's and other CNS pathologies. J Neurosci 28:12255-12267.

Kreutzberg GW (1996) Microglia: a sensor for pathological events in the CNS. Trends Neurosci 19:312-318.

Kuhnast B, Damont A, Hinnen F, Catarina T, Demphel S, Le Helleix S, Coulon C, Goutal S, Gervais P, Dollé F (2012) $\left[{ }^{18} \mathrm{~F}\right] \mathrm{DPA}-714,\left[{ }^{18} \mathrm{~F}\right] \mathrm{PBR} 111$, $\left[{ }^{18} \mathrm{~F}\right]$ FEDAA1106 - selective radioligands for imaging TSPO $18 \mathrm{kDa}$ with PET: automated radiosynthesis on a TRACERLAb FX-FN synthesizer and quality controls. Appl Radiat Isot 70:489-497.

Kutzelnigg A, Lucchinetti CF, Stadelmann C, Brück W, Rauschka H, Bergmann M, Schmidbauer M, Parisi JE, Lassmann H (2005) Cortical demyelination and diffuse white matter injury in multiple sclerosis. Brain 128:2705-2712.

Kutzelnigg A, Faber-Rod JC, Bauer J, Lucchinetti CF, Sorensen PS, Laursen H, Stadelmann C, Brück W, Rauschka H, Schmidbauer M, Lassmann H (2007) Widespread demyelination in the cerebellar cortex in multiple sclerosis. Brain Pathol 17:38-44.

Lopez-Diego RS, Weiner HL (2008) Novel therapeutic strategies for multiple sclerosis-a multifaceted adversary. Nat Rev Drug Discov 7:909-925.

Martín A, Boisgard R, Thézé B, Van Camp N, Kuhnast B, Damont A, Kassiou M, Dollé F, Tavitian B (2010) Evaluation of the PBR/TSPO radioligand $\left[{ }^{18} \mathrm{~F}\right]$ DPA-714 in a rat model of focal cerebral ischemia. J Cereb Blood Flow Metab 30:230-241.

Mattner F, Katsifis A, Staykova M, Ballantyne P, Willenborg DO (2005) Evaluation of a radiolabelled peripheral benzodiazepine receptor ligand in the central nervous system inflammation of experimental autoimmune encephalomyelitis: a possible probe for imaging multiple sclerosis. Eur J Nucl Med Mol Imaging 32:557-563.

Mattner F, Mardon K, Katsifis A (2008) Pharmacological evaluation of $\left[{ }^{123}\right.$ I]CLINDE: a radioiodinated imidazopyridine-3-acetamide for the study of peripheral benzodiazepine binding sites (PBBS). Eur J Nucl Med Mol Imaging 35:779-789.

Metz I, Lucchinetti CF, Openshaw H, Garcia-Merino A, Lassmann H, Freedman MS, Atkins HL, Azzarelli B, Kolar OJ, Brück W (2007) Autologous haematopoietic stem cell transplantation fails to stop demyelination and neurodegeneration in multiple sclerosis. Brain 130:1254-1262.

Minagar A, Shapshak P, Fujimura R, Ownby R, Heyes M, Eisdorfer C (2002) The role of macrophage/microglia and astrocytes in the pathogenesis of three neurologic disorders: HIV-associated dementia, Alzheimer disease, and multiple sclerosis. J Neurol Sci 202:13-23.

Nelson PT, Soma LA, Lavi E (2002) Microglia in diseases of the central nervous system. Ann Med 34:491-500.

Radu CG, Shu CJ, Shelly SM, Phelps ME, Witte ON (2007) Positron emission tomography with computed tomography imaging of neuroinflammation in experimental autoimmune encephalomyelitis. Proc Natl Acad Sci U S A 104:1937-1942.

Raivich G, Banati R (2004) Brain microglia and blood-derived macrophages: molecular profiles and functional roles in multiple sclerosis and animal models of autoimmune demyelinating disease. Brain Res Brain Res Rev 46:261-281.

Rock RB, Gekker G, Hu S, Sheng WS, Cheeran M, Lokensgard JR, Peterson PK (2004) Role of microglia in central nervous system infections. Clin Microbiol Rev 17:942-964, table of contents.

Sanders P, De Keyser J (2007) Janus faces of microglia in multiple sclerosis. Brain Res Rev 54:274-285.

Stadelmann C, Brück W (2008) Interplay between mechanisms of damage and repair in multiple sclerosis. J Neurol 255 [Suppl 1]:12-18.

Stankoff B, Wang Y, Bottlaender M, Aigrot MS, Dolle F, Wu C, Feinstein D, Huang GF, Semah F, Mathis CA, Klunk W, Gould RM, Lubetzki C, Zalc B (2006) Imaging of CNS myelin by positron-emission tomography. Proc Natl Acad Sci U S A 103:9304-9309.

Stepaniak JA, Gould KE, Sun D, Swanborg RH (1995) A comparative study of experimental autoimmune encephalomyelitis in Lewis and DA rats. J Immunol 155:2762-2769.

Turkheimer FE, Banati RB, Moran L, Duke D, Graeber MB (2004) Translating the cellular neuropathology of microglia into neuroimaging results. Neuropathol Appl Neurobiol 30 [Suppl 415[:1-4.

Vellinga MM, Oude Engberink RD, Seewann A, Pouwels PJ, Wattjes MP, van der Pol SM, Pering C, Polman CH, de Vries HE, Geurts JJ, Barkhof F (2008) Pluriformity of inflammation in multiple sclerosis shown by ultra-small iron oxide particle enhancement. Brain 131:800-807.

Winkeler A, Boisgard R, Martin A, Tavitian B (2010) Radioisotopic imaging of neuroinflammation. J Nucl Med 51:1-4.

Zeis T, Graumann U, Reynolds R, Schaeren-Wiemers N (2008) Normalappearing white matter in multiple sclerosis is in a subtle balance between inflammation and neuroprotection. Brain 131:288-303. 